\title{
INDICADORES DE QUALIDADE DA ASSISTÊNCIA: OPINIÃO DE ENFERMEIROS GERENTES DE HOSPITAIS DE ENSINO*
}

\author{
Mariana Angela Rossaneis ${ }^{1}$, Carmen Silvia Gabriel ${ }^{2}$, Maria do Carmo Lourenço Haddad ${ }^{3}$, Marcia Regina Antonietto da Costa \\ Melo $^{2}$, Andrea Bernardes ${ }^{2}$
}

${ }^{1}$ Enfermeira. Doutoranda em Enfermagem. Docente do Departamento de Enfermagem da Universidade Estadual de Londrina. Londrina, PR, Brasil.

${ }^{2}$ Enfermeira. Doutora em Enfermagem. Docente do Departamento de Enfermagem da Escola de Enfermagem de Ribeirão Preto. Ribeirão Preto, SP, Brasil.

${ }^{3}$ Enfermeira. Doutora em Enfermagem. Docente do Departamento de Enfermagem da Universidade Estadual de Londrina. Londrina, PR, Brasil.

RESUMO: Estudo descritivo de abordagem quantitativa que teve como objetivo identificar a opinião de enfermeiros gerentes de hospitais de ensino sobre a pertinência de indicadores de qualidade para gerenciar os serviços de enfermagem. A população foi composta por nove gestores dos serviços de enfermagem de hospitais de ensino do estado do Paraná. Os dados foram coletados entre os meses de agosto e setembro de 2012, com um questionário eletrônico referente à caracterização dos enfermeiros e sua opinião sobre os indicadores de qualidade. Os participantes desse estudo valorizaram os indicadores assistenciais para gerenciamento dos serviços de enfermagem e avaliação da qualidade assistencial em detrimento dos indicadores institucionais gerais e de gestão de pessoas. O gerenciamento das instituições de saúde exige dos profissionais competências para analisar o processo de trabalho e tomar decisões que visem à melhoria contínua da assistência e a competitividade das instituições.

DESCRITORES: Indicadores de qualidade em assistência à saúde; Qualidade da assistência à saúde; Avaliação em enfermagem; Enfermagem.

\section{HEALTH CARE QUALTTY INDICATORS: THE OPINION OF NURSING MANAGERS OF TEACHING HOSPITALS}

ABSTRACT: Quantitative descriptive study aimed to identify the opinion of nursing managers of teaching hospitals on the relevance of quality indicators in the management of nursing services. The population was composed of nine nursing managers of teaching hospitals of the state of Paraná. Data were collected in the months of August and September 2012, through a tool - a questionnaire on quality indicators made available electronically to be completed by the nurses. The participants showed greater appreciation of quality indicatorsfor the management of nursing services and evaluation of health care than to general indicators and personnel management indicators.For the management of health institutions, professional must be able to analyze work processes and make decisions aimed to the continuous improvement of care and competitiveness of institutions.

DESCRIPTORS: Health care quality indicators; Health care quality; Nursing evaluation; Nursing.

\section{INDICADORES DE CUALIDAD DE LA ASISTENCIA: OPINIÓN DE ENFERMEROS GERENTES DE HOSPITALES DE ENSEÑANZA}

RESUMEN: Estudio descriptivo de abordaje cuantitativo que tuvo como objetivo identificar la opinión de enfermeros gerentes de hospitales de enseñanza sobre la pertinencia de indicadores de cualidad para gerenciar los servicios de enfermería. La población fue compuesta por nueve gestores de los servicios de enfermería de hospitales de enseñanza del estado de Paraná. Los datos fueron obtenidos entre los meses de agosto y septiembre de 2012, con un cuestionario electrónico acerca de la caracterización de los enfermeros y su opinión sobre los indicadores de cualidad. Los participantes de ese estudio valoraron los indicadores asistenciales para gerenciamento de los servicios de enfermería y evaluación de la cualidad asistencial en detrimento de los indicadores institucionales generales y de gestión de personas. El gerenciamento de las instituciones de salud exige de los profesionales competencias para analizar el proceso de trabajo y tomar decisiones para obtener la mejoría continua de la asistencia y la competitividad de las instituciones.

DESCRIPTORES: Indicadores de cualidad en asistencia a la salud; Cualidad de la asistencia a la salud; Evaluación en enfermería; Enfermería.

*Artigo extraído de dissertação, intitulada: “Indicadores de qualidade adotados nos serviços de enfermagem de hospitais de ensino do Estado do Paraná, Brasil." Escola de Enfermagem de Ribeirão Preto, 2013.

Autor Correspondente:

Mariana Angela Rossaneis

Universidade Estadual de Londrina

Av. Brasília, 1100 - 86600-000 - Rolândia, PR, Brasil

E-mail: marianarossaneis@gmail.com
Recebido: $11 / 06 / 2015$

Finalizado: 22/10/2015 


\section{INTRODUÇÃO}

A melhoria contínua da qualidade assistencial requer avaliações sistemáticas dos cuidados prestados aos usuários dos serviços de saúde, visando à identificação dos fatores que interferem no processo de trabalho dos profissionais envolvidos na assistência.

As gerências das instituições de saúde estão adotando métodos de avaliação dos seus serviços com base em indicadores para tomar decisões na busca da melhoria de seus processos e resultados. A enfermagem, como parte fundamental e de grande relevância nas instituições hospitalares, deve instrumentalizar-se de ferramentas que avaliem o desempenho e a qualidade dos seus serviços e identifiquem oportunidades de melhorias seus resultados ${ }^{(1)}$.

Os indicadores são utilizados para medição do desempenho de funções, sistemas ou processos, ou seja, um valor estatístico que indica o alcance de metas ao longo do tempo ${ }^{(2)}$. Os indicadores são construídos em sua maioria mediante uma expressão matemática, em que o numerador representa o total de eventos predefinidos e o denominador, a população de risco selecionada, observando-se a confiabilidade, a validade, a sensibilidade, e especificidade e o valor preditivo dos dados ${ }^{(3)}$.

Os indicadores podem constituir instrumento de força e poder para mudar processos internos de trabalho, quando a coleta e os resultados são tratados adequadamente. Contudo, a seleção de indicadores para o processo avaliativo deve levar em consideração aspectos como as políticas assistenciais, educacionais e gerenciais em saúde; a missão e a estrutura organizacional; os programas e as propostas de trabalho das instituições de saúde; os recursos humanos, materiais, financeiros e físicos disponíveis e as expectativas da clientela atendida ${ }^{(4)}$.

Os indicadores de qualidade da equipe de enfermagem permitem a compreensão de fenômenos complexos, tornando-os quantificáveis, de maneira que possam ser analisados em conjunto com outros indicadores para a compreensão do processo de trabalho e se os objetivos almejados estão sendo alcançados ${ }^{(5)}$.

Estudos sobre indicadores que avaliam a qualidade e desempenho dos serviços de enfermagem vêm ganhando destaque nos modelos de avaliação. Pesquisa ${ }^{(6)}$ identificou, por meio de revisão sistemática de literatura nacional e internacional, 77 indicadores utilizados para análise da qualidade dos serviços de enfermagem e os classificou segundo a teoria de Donabedian, sendo que $74 \%$ dos indicadores foram classificados como de processo, $16,6 \%$ como sendo de estrutura e 9,4\% como indicadores de resultado.

A utilização de indicadores que avaliam a assistência de enfermagem é essencial para o gerenciamento dos serviços de saúde, uma vez que permite o monitoramento dos resultados da assistência prestada ao usuário e proporciona a identificação de necessidades de intervenções para melhoria do desempenho, produtividade e qualidade dos serviços.

Sendo assim, este estudo tem como objetivo identificar a opinião de enfermeiros gerentes de hospitais de ensino sobre a pertinência de indicadores de qualidade para gerenciar os serviços de enfermagem.

\section{MÉTODO}

Trata-se de um estudo exploratório, descritivo, transversal de abordagem quantitativa. O local da pesquisa constitui-se de nove hospitais gerais de ensino de alta complexidade, localizados no Estado do Paraná. No período de coleta de dados, o estado do Paraná possuía 15 hospitais de ensino, sendo que treze são categorizados como hospitais gerais. Foram convidados todos os enfermeiros gerentes dos serviços de enfermagem das instituições e nove aceitaram participar da pesquisa.

A coleta de dados foi realizada entre os meses de agosto e setembro de 2012, com um questionário eletrônico elaborado no programa Google Drive ${ }^{\circledast}$. O link de acesso ao questionário foi enviado aos participantes do estudo via correio eletrônico. O questionário continha dados referentes à caracterização dos enfermeiros (sexo, idade, tempo de formação profissional, tempo de experiência no gerenciamento dos serviços de enfermagem, tempo que possuem vínculo empregatício com a instituição em estudo e a realização de pós-graduação); e uma questão que permitia identificar a opinião dos enfermeiros sobre a pertinência dos indicadores de qualidade da assistência de enfermagem, por meio de uma Escala de Likert de quatro itens: muito pertinente, pertinente, pouco pertinente, não pertinente.

Os indicadores avaliados pela Escala Likert são os utilizados pelos hospitais que constituem o Núcleo de Apoio a Gestão Hospitalar, descritos 
na $1^{\mathrm{a}}$ e $2^{\mathrm{a}}$ edição do Manual de Indicadores Hospitalares publicado pelo Programa Compromisso com a Qualidade Hospitalarem 2012 e outros indicadores identificados na literatura considerados adequados para avaliar a qualidade dos serviços de enfermagem ${ }^{(1,7)}$. Esses indicadores foram subdivididos em indicadores institucionais gerais, indicadores assistenciais e indicadores de gestão.

Os indicadores institucionais gerais incluíram: média de permanência hospitalar, taxa de ocupação hospitalar, taxa de infecção hospitalar e taxa de mortalidade. Os indicadores assistenciais foram compostos por: incidência de queda do paciente, incidência de extubação acidental, perda de sonda nasogastroenteral, incidência de Úlcera Por Pressão (UPP), incidência de flebite, incidência de obstrução de cateter venoso central, incidência de não conformidade na administração de medicamentos, satisfação do cliente com os serviços de enfermagem, não conformidade nos registros de enfermagem. $E$ os indicadores de gestão de pessoas avaliados foram: distribuição de enfermeiros / leito, distribuição de técnico/ auxiliares de enfermagem por leito, taxa de absenteísmo de enfermagem, taxa de rotatividade de enfermagem, taxa de acidente de trabalho de profissionais de enfermagem e horas de treinamento de profissionais de enfermagem ${ }^{(1,7)}$.

Este estudo foi submetido ao Comitê de Ética em Pesquisa da Universidade Estadual de Londrina e aprovado sob o parecer $\mathrm{n}^{\circ} 84178$ e CAAE 04053112.0.0000.5231.

\section{RESULTADOS}

Dentre os participantes do estudo, sete eram do sexo feminino e a idade variou entre 28 e 62 anos, média de 45 anos. O tempo de formação oscilou de oito a 35 anos, média de 21 anos. Em relação ao tempo que possuem vínculo empregatício com o hospital, houve uma variação de quatro a 26 anos, com média de 15 anos. Todos os enfermeiros haviam atuado na assistência de enfermagem antes de assumirem o cargo atual e apenas três possuíam experiência na área de gestão.

Os enfermeiros eram pós-graduados, sendo que nove realizaram curso de especialização nível lato sensu e quatro stricto sensu. As áreas dos cursos de pós-graduação citados pelos participantes do estudo foram: administração hospitalar, gerenciamento dos serviços de enfermagem, saúde pública, saúde da mulher, assistência de enfermagem em saúde do adulto, saúde do trabalhador, enfermagem em centro cirúrgico e central de materiais e gestão estratégica de recursos humanos.

A opinião dos gerentes dos serviços de enfermagem das instituições em estudo sobre a pertinência dos indicadores institucionais gerais, indicadores assistenciais e indicadores de gestão de pessoas para avaliar a qualidade da assistência de enfermagem está apresentada na Tabela 1.

Verifica-se na Tabela 1 que os indicadores incidência de úlcera por pressão e incidência de não conformidade na administração de medicamentos foram considerados por $100 \%$ dos entrevistados como indicadores muito pertinentes para avaliar a qualidade da assistência de enfermagem.

Os indicadores incidência de queda do paciente, incidência de flebite, incidência de obstrução de cateter venoso central, não conformidade nos registros de enfermagem, taxa de infecção hospitalar, horas de treinamento de profissionais de enfermagem, taxa de acidente de trabalho de profissionais de enfermagem, taxa de rotatividade de enfermagem e satisfação do cliente com a enfermagem também foram considerados pela maioria indicadores de enfermagem muito pertinentes para avaliar o cuidado.

Já os indicadores incidência de extubação não programada, perda de sonda NGE, taxa de ocupação hospitalar, taxa de mortalidade hospitalar, distribuição de enfermeiros/ leito, distribuição de técnicos e auxiliares de enfermagem/leito e taxa de absenteísmo de enfermagem foram considerados apenas pertinentes para avaliar a enfermagem. Ainda, o indicador médio de permanência hospitalar foi considerado pertinente e pouco pertinente pelos entrevistados.

\section{DISCUSSÃO}

Identificou-se que a maioria dos enfermeiros gerentes que responderam $\mathrm{o}$ instrumento de pesquisa era do sexo feminino e a média de idade foi de 43 anos. Este fato tem relação com os aspectos históricos da profissão, considerando a predominância do gênero feminino. A maior parte dos entrevistados possuía entre 21 a 30 anos de formados e possuía vínculo empregatício de mais de 10 anos com a instituição. Todos os entrevistados haviam atuado na assistência direta ao paciente antes de o cargo atual. Apenas três gerentes relataram que possuíam experiência na área de gestão hospitalar antes de assumiram o 
Tabela 1 - Opinião dos enfermeiros sobre indicadores de qualidade utilizados no gerenciamento dos serviços de enfermagem. Londrina, PR, Brasil, 2012

\begin{tabular}{|c|c|c|c|c|c|c|c|c|}
\hline \multirow[t]{2}{*}{$\begin{array}{l}\text { Indicadores de qualidade da assistência de } \\
\text { enfermagem }\end{array}$} & \multicolumn{2}{|c|}{$\begin{array}{c}\text { Muito } \\
\text { Pertinente }\end{array}$} & \multicolumn{2}{|c|}{ Pertinente } & \multicolumn{2}{|c|}{$\begin{array}{l}\text { Pouco } \\
\text { Pertinente }\end{array}$} & \multicolumn{2}{|c|}{$\begin{array}{l}\text { Não } \\
\text { Pertinente }\end{array}$} \\
\hline & $\mathbf{n}$ & $\%$ & $\mathbf{n}$ & $\%$ & $\mathbf{n}$ & $\%$ & $\mathbf{n}$ & $\%$ \\
\hline \multicolumn{9}{|l|}{ Indicadores Institucionais Gerais } \\
\hline Taxa de infecção hospitalar & 7 & 77,8 & 2 & 22,2 & - & - & - & - \\
\hline Taxa de mortalidade hospitalar & 4 & 44,4 & 5 & 55,6 & - & - & - & - \\
\hline Taxa de ocupação hospitalar & 3 & 33,3 & 4 & 44,5 & 1 & 11,1 & 1 & 11,1 \\
\hline Média de permanência hospitalar & 2 & 22,3 & 3 & 33,3 & 3 & 33,3 & 1 & 11,1 \\
\hline \multicolumn{9}{|l|}{ Indicadores Assistenciais } \\
\hline Incidência de flebite & 7 & 77,8 & 2 & 22,2 & - & - & - & - \\
\hline $\begin{array}{l}\text { Incidência de obstrução de cateter venoso } \\
\text { central }\end{array}$ & 6 & 66,7 & 3 & 33,3 & - & - & - & - \\
\hline Incidência de extubação não programada & 2 & 22,2 & 5 & 55,6 & 2 & 22,2 & - & - \\
\hline Perda de sonda NGE & 2 & 22,2 & 4 & 44,5 & 3 & 33,3 & - & - \\
\hline $\begin{array}{l}\text { Não conformidades nos registros de } \\
\text { Enfermagem }\end{array}$ & 5 & 55,6 & 3 & 33,3 & 1 & 11,1 & - & - \\
\hline $\begin{array}{l}\text { Horas de treinamento de Profissionais de } \\
\text { Enfermagem }\end{array}$ & 7 & 77,8 & 2 & 22,2 & - & - & - & - \\
\hline $\begin{array}{l}\text { Taxa de acidente de trabalho de } \\
\text { profissionais de enfermagem }\end{array}$ & 7 & 77,8 & 2 & 22,2 & - & - & - & - \\
\hline Taxa de rotatividade de enfermagem & 5 & 55,6 & 3 & 33,3 & - & - & 1 & 11,1 \\
\hline \multicolumn{9}{|l|}{ Indicadores de Gestão de Pessoas } \\
\hline Distribuição enfermeiros/leito & 4 & 44,4 & 5 & 55,6 & - & - & - & - \\
\hline $\begin{array}{l}\text { Distribuição de técnicos e auxiliares de } \\
\text { enfermagem/leito }\end{array}$ & 4 & 44,4 & 5 & 55,6 & - & - & - & - \\
\hline Taxa de absenteísmo de enfermagem & 3 & 33,3 & 4 & 44,5 & 2 & 22,2 & - & - \\
\hline Satisfação do cliente com a enfermagem & 6 & 66,7 & 3 & 33,3 & - & - & - & - \\
\hline
\end{tabular}

cargo atual. O tempo de carreira na instituição e a capacitação técnica do enfermeiro são fatores que interferem na escolha do profissional que assume um cargo gerencial. Na área da saúde, esses prérequisitos, muitas vezes são mais considerados na escolha do gestor, do que a capacitação para o exercício da função gerencial ${ }^{(8)}$.

Ainda, verifica-se no estudo que todos os entrevistados possuíam certificação lato sensu e sete haviam realizado especializações relacionadas à área de gestão em saúde. Esses resultados podem estar relacionados ao fato dos entrevistados atuarem em hospitais de ensino vinculados a instituições de ensino superior. Além disso, após a especialização, grande parte dos profissionais de enfermagem tem optado por realizar cursos stricto sensu, que são de natureza acadêmica e de pesquisa. Observa-se também que a pós-graduação caracterizada por formar um estrato intelectual superior, tem atraído os enfermeiros na busca do status e destaque entre profissionais ${ }^{(8)}$.
Osindicadoresincidência de nãoconformidade na administração de medicamentos e incidência de UPP foram considerados por todos os participantes do estudo muito pertinentes para avaliar a assistência de enfermagem. A incidência de não conformidade na administração de medicamentos é um dos indicadores que está mais relacionado à rotina da equipe de enfermagem. A prática da administração de medicamentos em uma organização hospitalar é um sistema complexo, com vários processos interligados, interdependentes e constituídos por profissionais de diferentes áreas do conhecimento $^{(9)}$. $\mathrm{O}$ estabelecimento e monitoramento de indicadores associados a problemas relacionados a medicamentos visam assegurar a terapêutica necessária e a recuperação do usuário. Os resultados dessa avaliação são imprescindíveis para que as falhas sejam evitadas e, principalmente, prevenidas.

A equipe de enfermagem também deve desenvolver estratégias para a prevenção das 
UPP atuando no controle dos fatores de risco como pressão, cisalhamento, fricção, umidade, nutrição, capacidade de movimentação. Por esse motivo, a utilização de indicadores de UPP tem sido associada a um cuidado com qualidade ${ }^{(10)}$.

Já os indicadores incidência de queda do paciente, incidência de flebite, incidência de obstrução de cateter venoso central e não conformidades nos registros de enfermagem fazem referência aos processos assistenciais específicos da equipe de enfermagem, os quais estão estreitamente relacionados às tarefas desenvolvidas diariamente pelos profissionais e são capazes de retratar a qualidade desses processos. A queda do paciente consiste num dos principais indicadores de resultado e é definido também como um evento-sentinela. A avaliação do risco de queda bem como a sua prevenção devem ser incorporadas na prática cotidiana da enfermagem ${ }^{(11)}$.

A flebite também é uma das complicações mais frequentes da terapia intravenosa e é definida como a inflamação de uma veia. Especialistas recomendam que a prevalência desse evento deva ser menor que $5 \%{ }^{(12)}$. Levandose em consideração que a maioria dos pacientes hospitalizados necessita de um acesso venoso, é possível avaliar a importância de um indicador específico relacionado a essa complicação.

Apesar do indicador incidência de obstrução de cateter venoso central ter sido considerado como muito pertinente para avaliar a qualidade de enfermagem, uma pesquisa que analisou a administração de medicamentos intravenosos em um hospital público, identificou que em $86,4 \%$ dos procedimentos de administrações de medicamentos por via intravenosa, não era observada a permeabilidade do cateter ${ }^{(13)}$.

A avaliação da permeabilidade deve ser realizada com o intuito de verificar se o cateter está pérvio ou se ocorreu a formação de trombo em suas extremidades, o que pode ser feito checando o fluxo da solução infundida ${ }^{(13)}$. Fica evidente a importância do conhecimento por parte da equipe de enfermagem sobre os mecanismos que envolvem a instalação e manutenção do acesso venoso que possibilite segurança ao paciente e a preservação e detecção precoce de possíveis complicações ${ }^{(1)}$.

Ainda na gerência do cuidado, observa-se que as anotações de enfermagem constituem um indicador fundamental na avaliação da qualidade assistencial $^{(14)}$. Entretanto, esse indicador tem sido pouco utilizado pelas instituições de saúde, uma vez que a análise das anotações de enfermagem exige a estruturação de um serviço de auditoria nos serviços de saúde. Sendo assim, muitas vezes a dificuldade de recursos financeiros e/ou a falta de interesse em avaliar os seus serviços fazem com que esse indicador seja pouco utilizado.

Salienta-se que esse indicador contribui para a continuidade da assistência ao paciente, uma vez que esses registros devem traduzir o máximo de conhecimento sobre as condições de saúde dos indivíduos, incluindo-se tanto os aspectos referentes a procedimentos quanto as necessidades, queixas e evolução dos pacientes $^{(14)}$. Além disso, a identificação nas falhas dos registros reforça a necessidade de educação permanente com a equipe, visando à melhoria na comunicação escrita e ainda, quando necessário, maior esclarecimento em ações judiciais.

Observa-se que os enfermeiros participantes desse estudo valorizaram os indicadores assistenciais para gerenciamento dos serviços de enfermagem e avaliação da qualidade assistencial em detrimento aos indicadores institucionais gerais e indicadores de gestão de pessoas. Esses achados também foram encontrado em estudo que identificou a opinião de enfermeiros de um hospital geral do interior do Estado de São Paulo, sobre os indicadores de enfermagem indicadores dos processos assistenciais específicos da equipe de enfermagem ${ }^{(1)}$.

Apenas dois enfermeiros consideraram os indicadores perda de sonda NGE e incidência de extubação não programada muito pertinentes, a maioria afirmou ser apenas pertinente ou não pertinente para avaliar a assistência de enfermagem. Apesar de esses indicadores estarem relacionados diretamente aos cuidados com o paciente $^{(1,9,15)}$, infere-se que esse resultado pode estar associado ao fato desses eventos ocorrerem devido ao quadro psicológico do paciente e/ou sua manipulação por outros profissionais que também prestam assistência como médicos, fisioterapeutas, etc.

A taxa de infecção hospitalar foi o único indicador considerado muito pertinente para avaliar a enfermagem pela maioria dos enfermeiros pesquisados. Este indicador foi considerado de maior importância em estudo que analisou a opinião dos enfermeiros sobre a utilização de indicadores de qualidade para avaliação da assistência de enfermagem ${ }^{(9)}$. Os resultados relacionados à opinião dos entrevistados sobre esse indicador demonstram que os enfermeiros 
percebem a necessidade de desenvolverem uma visão mais abrangente e multidisciplinar sobre os resultados da assistência prestada, o que permite avaliar o cuidado e implementar ações de melhoria da qualidade assistencial ${ }^{(15)}$.

A taxa de mortalidade e a taxa de ocupação hospitalar foram consideradas indicadores pertinentes. Ainda, a média de permanência hospitalar foi referida como pertinente e pouco pertinente pela maioria dos participantes. Indicadores globais de resultado assistencial tradicional como a taxa de ocupação hospitalar, taxa de mortalidade e média de permanência são analisados pela direção do hospital, fora do rol daqueles específicos da equipe de enfermagem ${ }^{(6)}$. Essa seria uma explicação do fato desses indicadores terem sido considerados pertinentes e não pertinentes para o gerenciamento dos serviços de enfermagem pelos entrevistados ou participantes doestudo, umavezqueaenfermagem ainda não os utiliza sistematicamente e não os compreende como relacionados aos resultados finais da assistência ao paciente. Contudo, devem ser olhados com um foco multidisciplinar, já que representam os resultados do trabalho de toda a equipe de saúde ${ }^{(6)}$.

Quanto aos indicadores relacionados à gestão de pessoas, a taxa de rotatividade de pessoal ou turnover foi reconhecida como um indicador de qualidade essencial para o gerenciamento de recursos humanos em estudo desenvolvido com enfermeiros de um hospital universitário. A rotatividade no quadro de profissionais representa uma dificuldade no gerenciamento de enfermagem e interfere diretamente qualidade do cuidado, pela perda de profissionais capacitados, e também nos custos para a organização ${ }^{(16)}$.

Os indicadores relacionados aos acidentes de trabalho de profissionais podem identificar as falhas no processo e na estrutura das unidades hospitalares, umavezqueasaltas taxas deacidentes são relacionadas à falha do profissional, técnica inadequada, deficiência do material utilizado, entre outros. Sendo assim, o monitoramento dos acidentes de trabalho nas instituições de saúde possibilita o reconhecimento e análise dos riscos ocupacionais nestes ambientes. Dessa forma, é possível evitar ou minimizar a ocorrência de acidentes de trabalho e desenvolver medidas de controle e prevenção, bem como a promoção da conscientização sobre as práticas seguras entre os profissionais ${ }^{(17)}$.

Em relação ao item horas de treinamento de profissionais de enfermagem, observa-se que a garantia de capacitação dos profissionais na instituição de saúde, bem como a monitoração constante pós-capacitação, é necessária para a promoção de assistência de qualidade dentro dos padrões estabelecidos. A quantidade de horas dedicadas à capacitação da equipe está diretamente relacionada ao melhor preparo desta para a assistência ${ }^{(1)}$.

Entretanto, o gerenciamento de indicadores de treinamento demonstra que os índices são favoráveis para a avaliação quantitativa dos dados, porém ainda é um desafio para a enfermagem desenvolver uma avaliação que envolva os resultados qualitativos relacionados à mudança de comportamento e atitudes na prática assistencial.

As taxas de distribuição de trabalhadores por leito podem ser consideradas indicadores de estrutura de serviços de saúde e estão diretamente relacionadas à qualidade da assistência prestada ao paciente ${ }^{(15)}$. Estabelecer um adequado dimensionamento do quadro de enfermagem é fundamental para se garantir uma assistência de qualidade, pois estabelece o quantitativo de pessoal necessário para atender à demanda assistencial da clientela atendida ${ }^{(16)}$.

Nessa perspectiva, é imprescindível que os gerentes de enfermagem monitorem o índice de ausências dos profissionais de enfermagem como um indicador de gestão de recursos humanos, e utilizem um quantitativo de pessoal adicional para cobertura dessas ausências, evitando a sobrecarga de trabalho e, consequentemente, a elevação do absenteísmo dos trabalhadores, que interfere, diretamente, na segurança e qualidade da assistência prestada ${ }^{(18-19)}$.

O indicador satisfação do cliente com a enfermagem foi apontado como muito pertinente paraavaliaros serviços deenfermagem. Asatisfação é uma avaliação pessoal do serviço recebido, fundamentada em padrões subjetivos de ordem cognitivo-afetivo dos usuários. Há quatro grupos principais determinantes da satisfação, que são: a característica do paciente (cliente), as condições sociodemográficas, as expectativas destes sobre o atendimento médico e de enfermagem, e as características dos profissionais que prestam o atendimento, incluindo traços de personalidade e qualidade técnica ${ }^{(9)}$.

A satisfação do usuário diz respeito a atender às necessidades do usuário, concedendo-lhe um tratamento individualizado e humanizado. Esse indicador fornece informações importantes para melhoria da assistência dos serviços de saúde. 


\section{CONCLUSÃO}

Nesse estudo foi possível identificar que os gerentes dos serviços de enfermagem conhecem os indicadores de qualidade e os consideram importantes para o gerenciamento do cuidado assistencial. O gerenciamento das instituições de saúde exige dos profissionais competências para analisar o processo de trabalho e tomar decisões que visem à melhoria contínua da assistência e à competitividade das instituições. Para isso se faz necessária a avaliação periódica dos serviços fundamentada em indicadores para o alcance de padrões de qualidade.

É necessário estabelecer estrutura e rotina de coleta e análise dos indicadores para que os seus resultados sejam fidedignos à realidade das instituições. Pressupõe-se que a dificuldade na obtenção dos dados para análise por indicadores é uma das principais razões para que esses não sejam plenamente adotados pelo serviço. Contudo, a reflexão sobre o processo de trabalho fundamentado em informações válidas e legítimas é a única maneira de se obter melhores resultados.

\section{REFERÊNCIAS}

1. Silva CS, Gabriel CS, Bernardes A, Évora YDM. Opinião do enfermeiro sobre indicadores que avaliam a qualidade na assistência de enfermagem. Rev. GaúchaEnferm. 2009; 30(2):263-71.

2. Joint Commission International. Joint Commission International Accreditation Standards for Hospitals. [Internet] 2014 [acesso em 22 ago 2015]. Disponível: https://www.jcrinc.com/assets/1/14/JCIH14_Sample_ Pages.pdf.

3. Tronchin DMR, Melleiro MM, Takahasshi RT. A qualidade e a avaliação dos serviços de saúde e de enfermagem. In: Kurcgant $\mathrm{P}$, organizadora. Gerenciamento em enfermagem. $2^{a}$ ed. Rio de Janeiro: Guanabara Koogan; 2010. p.77-107.

4. Fusco SFB, Spiri WC. Análise dos indicadores de qualidade de centros de material e esterilização de hospitais públicos acreditados. TextoContextoEnferm. 2014; 23(2):426-33.

5. Haddad MCFL, Rossaneis MA. Indicadores de qualidade da assistência de enfermagem. In: Vale EG, Lima JR, Felli VEA. Programa de atualização em enfermagem. Brasília: Artmed; 2011. p.41-90.

6. Caldana G, Gabriel CS, Bernardes A, Évora YDM. Indicadores de desempenho em serviço de enfermagem hospitalar: revisão integrativa.Rev. Rene. 2011; 12(1):189-97.
7. Programa Compromisso com a qualidade hospitalar $(\mathrm{CQH})$. Manual de Indicadores de Enfermagem do Núcleo de Apoio a Gestão Hospitalar (NAGEH). $3^{\mathrm{a}}$ ed. São Paulo: Van Moorsel; 2012. p.60.

8. Furukawa PO, Cunha ICKO. Perfil e competências de gerentes de enfermagem de hospitais acreditados. Rev. Latino-Am. Enfermagem. 2011; 19(1):106-14.

9. Cintra EA, Pinto AC, Sousa EO, Rosa EV, Lima IA, Rodrigues SO. Utilização de indicadores de qualidade para avaliação da assistência de enfermagem: opinião dos enfermeiros. J. Health Sci. Inst. 2010; 28(1):29-34.

10. Vargas e Silva MR, Dick NRM, Martini AC. Incidência de úlcera por pressão como indicador de qualidade na assistência de enfermagem. REUFSM. 2012;2(2):339-46.

11. Inoue KC, Matsuda LM, Melo WA, Murassaki ACY, Hayakawa LY. Risco de queda da cama. O desafio da enfermagem para a segurança do paciente. Invest. Educ. Enferm. 2011; 29(3):459-66.

12. Lopes PC, Ventramim P, Stramasso LV. Indicadores relacionados a flebite. In: Leão ER, Silva CPR, Alvarenga DC, Mendonça SHF. Qualidade em saúde e indicadores como ferramenta de gestão. São Caetano do Sul: Yendis; 2010. p. 221-231.

13. Silva LD, Camerini FG. Análise da administração de medicamentos intravenosos em hospital da rede sentinela. Texto Contexto Enferm. 2012; 21(3):633-41.

14. Silva JA, Grossi ACM, Haddad MCL, Marcon SS. Avaliação da qualidade das anotações de enfermagem em unidade semi-intensiva. Esc. Anna Nery. 2012; 16(3):577-82.

15. Gabriel CS, Melo MRAC, Rocha FLR, Bernardes A, Miguelaci T, Silva MLP. Utilização de indicadores de desempenho em serviço de enfermagem de hospital público. Rev. Latino-Am. Enfermagem. 2011; 19(5):124754.

16. Vieira APM, Kurcgant P. Indicadores de qualidade no gerenciamento de recursos humanos em enfermagem: elementos constitutivos segundo percepção de enfermeiros. Acta paul.enferm.2010; 23(1):11-15.

17. Bakke AH, Araujo NMC. Acidentes de trabalho com profissionais de saúde de um hospital universitário. Prod. 2010; 20(4):669-76.

18. Sancinetti TR, Soares AVN, Lima AFC, Santos NC, Melleiro MM, Fugulin FMT,et al. Taxa de absenteísmo da equipe de enfermagem como indicador de gestão de pessoas. Rev. esc. enferm. USP. 2011; 45(4):1007-12.

19. Faria JIL, Kurcgant P. Licenças saúde de profissionais de enfermagem: características e ações adotadas pelos enfermeiros-chefe. CogitareEnferm. 2014;19(2):224-31. 